\title{
Resignificación de la Estadística Aplicada a las Ciencias Sociales
}

Resignification of Statistics Applied to

Social Sciences

\section{Sergio Oscar Anchorena}

Ingeniero en Construcciones

(Universidad Nacional del Centro de la Provincia de Buenos Aires, Argentina)

Licenciado en Economía

(Universidad Nacional de Mar del Plata, Argentina)

Máster en Ciencias Sociales

(FLACSO, Argentina)

Magíster Scienteae en Epistemología y Metodología de la Ciencia

(Universidad Nacional de Mar del Plata, Argentina)

Máster en Economía y Desarrollo Territorial

(Universidad de Huelva, España)

Doctor en Filosofía y Ciencias de la Educación

(Universidad del País Vasco, España)

Doctor Área Ciencias Jurídicas y Sociales, programa Economía Social y Desarrollo Local

(Universidad de Huelva, España)

Correo electrónico: pollo_mdp@yahoo.com 


\title{
Resumen
}

En esta nota se hace una mirada retrospectiva y prospectiva de la disciplina Estadística Aplicada a las Ciencias Sociales, a la luz de los actuales desarrollos en la comprensión de los hechos sociales, y de los desarrollos instrumentales de la Informática Aplicada al análisis de datos. La Estadística, en tanto que "Ciencia de los Datos" debe adaptarse al análisis tanto de los datos que configuran las condiciones objetivas, como a aquellos que permiten inferir las disposiciones subjetivas de los actores que participan de un hecho social, y así dar cuenta de las condiciones de posibilidad que contribuyen al estudio de "lo que pasa" en la sociedad.

\section{Palabras clave}

Estadística, Ciencias Sociales, Condiciones de posibilidad, Hecho social.

\begin{abstract}
This note makes a retrospective and prospective look at the discipline of Statistics Applied to Social Sciences, in light of current developments in the understanding of social facts, and of the instrumental developments of Applied Informatics to data analysis. Statistics, as "Data Science" must adapt to the analysis of both the data that determine the objective conditions, and those that allow inferring the subjective dispositions of the actors who participate in a social fact, and thus account for the conditions of possibility that contribute to the study of "what happens" in society.
\end{abstract}

\section{Keywords}

Statistics, Social Sciences, Conditions of possibility, Social fact. 


\section{Introducción}

El término alemán Statistik, fue introducido originalmente por Gottfried Achenwall en 1749, se refería al análisis de datos del Estado, es decir, la "ciencia del Estado". En su origen, por tanto, la estadística estuvo asociada a los Estados, fue en el siglo XIX cuando el término estadística adquirió el significado de recolectar y clasificar datos. Hoy el uso de la estadística se ha extendido más allá de sus orígenes como un servicio al Estado o al gobierno. Personas y organizaciones usan la estadística para entender datos y tomar decisiones en organizaciones, en la producción científica, y en la intervención social.

A partir de la revolución científico tecnológica de fines del siglo XX y que todavía continúa el complejo teleinformático ha modificado radicalmente las formas de obtener y procesar datos, tanto como el propio concepto de dato.

Mientras que la estadística tradicionalmente se ocupaba de los datos en tanto que "cantidades", es decir del concepto de dato como una representación simbólica de un atributo o variable cuantitativa o cualitativa, sumado a la masificación del uso de computadoras y al desarrollo de software que permiten procesar datos de ambos tipos, extienden el concepto de estadística al procesamiento de otros datos, referidos a las disposiciones de los actores sociales, que antes quedaban fuera de su alcance por barreras de tipo tecnológicas.

Por otra parte, la tendencia en la enseñanza de la estadística para las ciencias sociales "sugiere que los estudiantes aprenden más cuando los profesores usan ejemplos concretos y problemas relevantes para ellos" (Abusabha y Woelfel, 2003:567). Se recomienda, además que, para contar con ejemplos concretos y problemas relevantes a los estudiantes, proponer actividades de aprendizaje que vinculen los métodos cuantitativos a los enfoques cualitativos, debido a que estos últimos son utilizados con mayor recurrencia en los planes de estudio de las licenciaturas en Ciencias sociales. En esta combinación de métodos encontramos "la recopilación de los ricos detalles descriptivos de los datos cualitativos se puede usar para cuantificar y generalizar los resultados" (Sechrest y Sidani, 1995:84). En ese sentido, este trabajo 
pretende hacer una propuesta de resignificación del concepto de Estadística en lo que respecta a su aplicación a las Ciencias Sociales, y, más específicamente una propuesta sobre qué es lo que debería aprenderse en las carreras de Ciencias Sociales.

\section{La Realidad Social es lo que pasa}

La relevancia de los datos, en la investigación social, está dada porque esos datos den cuenta de lo que pasa en una realidad compleja, configurada por múltiples determinaciones objetivas y mediadas por la subjetividad y la intersubjetividad de los actores sociales.

Es por eso que la Nueva Estadística, debe brindar herramientas para el análisis de datos que den cuenta las condiciones de posibilidad de los hechos sociales, esto es, las "Condiciones Objetivas", objeto de estudio tradicional de la Estadística (Variables Cuantitativas, Cualitativas, Categóricas) y de las "Disposiciones Subjetivas" (Creencias, Saberes, Valores, Temores, etc.), tradicionales del paradigma interpretativo que hoy, pueden ser analizadas con la ayuda de herramientas informáticas.

Por condiciones de posibilidad debe entenderse el conjunto de condiciones objetivas y disposiciones subjetivas necesarias (aunque cada una de ellas, no suficiente), coexistentes, para que algo ocurra en el mundo social, y no ocurra otra cosa.

En la figura 1 se muestra una representación de las condiciones de posibilidad que permiten dar cuenta de un hecho social. 


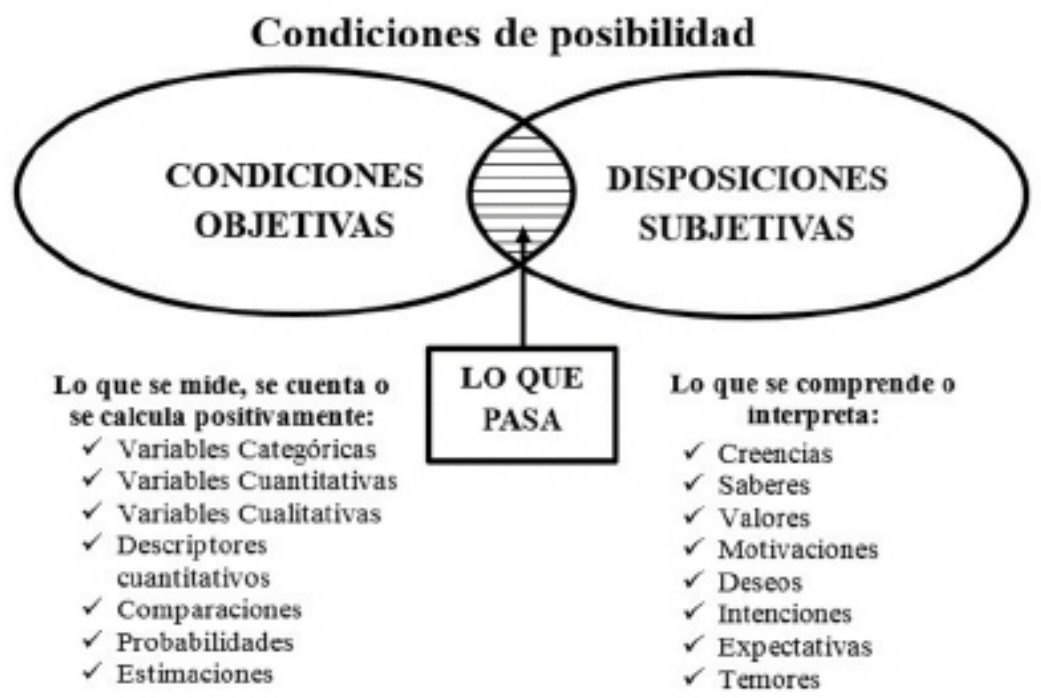

Figura 1: Las Condiciones de posibilidad de "lo social"

Fuente: Elaboración propia

Esta particularidad de los hechos sociales hace que, antes las mismas condiciones objetivas (de aquellos aspectos de la realidad que pueden verse, contarse o medirse, que enmarcan el hecho social), lo que pasa sea distinto, dependiendo de las disposiciones subjetivas, conscientes o inconscientes, manifiestas o no manifiestas, de les actores sociales que participan de "eso que está pasando".

Es importante entender que, si ante las mismas condiciones objetivas, desconociendo las subjetividades de los actores, siempre pasara lo mismo, eso no invalida el argumento de que las disposiciones subjetivas forman parte de las condiciones de posibilidad de aquello que está pasando. En este caso, desde un punto de vista instrumentalista, podría obviarse su análisis, pero, si lo que queremos es dar cuenta de aquello que pasó, o está pasando, esto es, investigar científicamente, las mismas deberían ser indagadas, inferidas y ponderadas en su debida importancia en la configuración de la realidad que se está indagando. 
Sin embargo, justamente lo más relevante del análisis de las disposiciones subjetivas, aparece en la explicación de lo distinto que ocurre cuando, aparentemente, desde el punto de vista de las condiciones objetivas, la realidad social es similar.

Esta visión de lo social, como el resultado de elementos objetivos y subjetivos, tiene su origen más elaborado en el famoso libro "La Construcción Social de la Realidad”, de Peter Berger y Thomas Luckmann, del año 1966, sin embargo, en esos días, la "ciencia de los datos" no contaba con los instrumentos de análisis necesarios para procesar los datos que hacen falta para el análisis de los componentes objetivos y subjetivos de esa "realidad social".

A modo de ejemplo, veamos un hecho actual, como la vacunación para inmunizar a la población contra el COVID-19, entre las condiciones objetivas necesarias para que esa vacunación ocurra están la existencia de vacunas, accesibles y disponibles para la población, la existencia de personal de la salud capacitado para aplicarlas, pero, si no existe la disposición subjetiva de les miembres de la población a vacunarse, la vacunación no ocurrirá. Lo mismo (no) ocurrirá, si, la población estuviera dispuesta a vacunarse, pero no hubiera vacunas ni personal de la salud.

Esto es importante a la hora de indagar y comprender los hechos sociales, pero tanto o más importante a la hora de plantear una intervención transformadora de lo social, ya que un diagnóstico, deberá incluir de la manera más exhaustiva las condiciones de posibilidad que dan cuenta de la situación actual y su transformación necesaria para lograr una mejora futura de esa situación.

Transformar la realidad puede implicar transformar las disposiciones subjetivas, las condiciones objetivas, o ambas, pero, para transformarlas, primero, hay que conocerlas. Y la Estadística, la Ciencia de los Datos, debe brindar las herramientas necesarias para analizar los datos que permiten aproximarse a esas condiciones y disposiciones.

\section{La Nueva Estadística Aplicada}

Tomando como punto de partida la definición de Estadística como la ciencia del análisis de datos, y la particularidad del conocimiento de las cien- 
cias sociales, que implican el análisis de las condiciones de posibilidad de los hechos sociales, desarrollada sucintamente en el apartado anterior, la Estadística Aplicada a las Ciencias Sociales, resulta la disciplina auxiliar de la Metodología y las técnicas de la Investigación Social, que se enfocan en el qué y cómo investigar, y en las fuentes y el instrumental para la recogida de datos.

$\mathrm{Al}$ respecto, entre las ventajas de combinar los métodos cuantitativos con los cualitativos en Ciencias Sociales se encuentra el que los investigadores y aprendices desarrollen la destreza para triangular resultados, lo que disminuye la falibilidad inherente a cada enfoque. Por ejemplo, los errores que puedan atribuirse a los datos de origen, dado que los métodos utilizan tipos de datos distintos, serán más confiables los resultados si proceden de una triangulación de métodos. Actualmente es común que, en la formación de profesionales de las Ciencias Sociales, en las diversas "asignaturas en las que se enseña a investigar" se ejercite el uso de los métodos de investigación cualitativos "introducir el razonamiento cuantitativo en los cursos sustanciales permite ligar el entrenamiento en métodos cuantitativos, especialmente estadísticos, con los asuntos medulares de las ciencias sociales" (Bridges, Gillmore, Pershing y Bates, 1998:24).

Así, además de los ya tradicionales contenidos de Estadística Descriptiva y Estadística Inferencial, la Nueva Estadística debe implicar, necesariamente, el uso de software, privilegiando el software libre o las licencias educativas, y el análisis de datos cuantitativos (de la estadística tradicional), incluyendo además el análisis de datos cualitativos, asistido por computadora.

$\mathrm{Al}$ respecto, existe una tradición en Argentina (tradición en la que yo mismo participo) del uso del Software Atlas.ti ${ }^{\circledR}$, que dispone de una versión gratuita para uso educativos. Si bien, el programa está diseñado para el marco teórico de análisis proporcionado por el Método Comparativo Constante (Grounded Theory, en su versión inglesa), el programa es versátil, y permite ser utilizado con otros marcos analíticos. Hay otros programas como MAXqda, Nud.ist ${ }^{\circledR}$ o Ethnograph ${ }^{\circledR}$ (Gibbs, 2014), pero a la hora de codificar y recuperar información, Atlas.ti ${ }^{\circledR}$ presenta numerosas ventajas que destacan, entre otres, Chernobilsky y D'Onofrio (2001), además de las contribuciones que estas autoras han hecho a su difusión a través de cursos de posgrado en 
diferentes Universidades Argentinas. Acaso la nota distintiva del Atlas.ti esté en la facilidad con la que permite visualizar redes semánticas, hipervínculos y codificaciones, en vista de red (Álvarez-Gayou Jurgenson, 2003 y Gibbs, 2014). También existen aplicaciones de R para este tipo de análisis, aunque la interfaz es bastante menos amigable.

Lo importante es que hoy, no puede separarse lo conceptual de la estadística, de lo instrumental del uso de computadoras, y el análisis de las condiciones objetivas (mal llamado metodología cuantitativa), del análisis de las disposiciones objetivas (mal llamado metodología cualitativa), que son imprescindibles, en conjunto, para dar cuenta de cualquier hecho social, y que ambos elementos deben estar presente desde el inicio de las prácticas investigativas en la formación de grado en todas las Ciencias Sociales.

\section{Referencias bibliográficas}

Abusabha, Rayane \& Woelfel, Mary (mayo 2003). Qualitative vs quantitative methods: Two opposites that make a perfect match. Journal of the American Dietetic Association 103 (5), 566569.

Álvarez-Gayou Jurgenson, Juan Luis (2003). Cómo hacer investigación cualitativa: Fundamentos y Metodología. México, Paidós Ecuador.

Berger, Peter, y Luckman, Tomas (1968). The Social Construction of Reatliy. New York, Random House.

Bridges, George; Gillmore, Gerald; Pershing, Jana; Bates, Kristin, (enero 1998). Teaching Quantitative Research Methods: A Quasi-Experimental Analysis. Teaching Sociology. American Sociological Association 26 (1), 14-28.

Chernobilsky, Lilia y D’Onofrio, María Guillermina, (2001). El análisis de datos cualitativos asistido por recursos informáticos: el software ATLAS/ti en un estudio sociológico sobre el papel social de la universidad pública argentina. Actas de las Terceras Jornadas sobre Etnografía y Métodos Cualitativos. Buenos Aires, Instituto de Desarrollo Económico y Social.

Gibbs, Graam (2014). El análisis de datos en la investigación cualitativa. Madrid, Ediciones Morata.

Sechrest, Lee \& Sidani, Souraya, (enero-marzo 1995). Quantitative and qualitative methods: Is There an Alternative. Evaluation and Program Planning 18 (1), 81-91.

Recibido: $18 / 02 / 2021$

Aceptado: 02/04/2021 